\title{
P03-8-2 Poster session
}

\section{Developing new therapies for neuronal ceroid lipofuscinoses or Batten disease}

\author{
Maija Dambrova ${ }^{1,2}$, Sara Mole $^{3}$

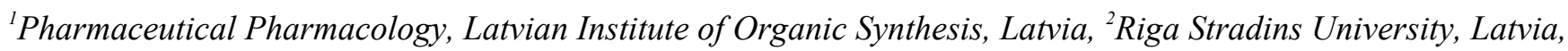 \\ ${ }^{3}$ University College London, UK
}

The neuronal ceroid lipofuscinoses (NCLs) or Batten disease is the most common of the rare neurodegenerative disorders of children, a group of rare inherited lysosomal storage disorders. They are severely debilitating and life-limiting neurodegenerative diseases beginning in childhood, with an estimated 14,000 affected world-wide, with about1400 new cases each year. The EU funded H2020 consortium BATCure aims to develop the first effective treatments for three genetically distinct NCL sub-types caused by mutations in intracellular transmembrane proteins: CLN3, CLN6 and CLN7 diseases. The goal of BATCure is to provide and test novel therapeutic leads, and to increase our understanding of the underlying biochemical and molecular basis of Batten disease. A comprehensive drug discovery effort is identifying compounds able to ameliorate the phenotype using high content imaging-based technology in cellular models of disease and follows the effects of small molecules on pathologically relevant phenotypes, such as expansion and distribution of lysosomal compartment and lysosomal storage. The use of automated screening and high throughput technology is an indispensable instrument in the pre-clinical phase of new drug discovery, especially accelerating drug development in diseases with no treatment available. A novel approach in BATCure is to use these approaches to screen our own intelligently designed compound libraries, as well as FDA collections of thousands of compounds to allow repositioning of known drugs as correctors of Batten disease. We will use efficacious compounds as a base for quantitative SAR modelling and the design of a novel set of new chemical entities for testing in a variety of cell and animal model systems. BATCure is also developing gene therapy approaches. In addition, BATCure provides a mechanism to involve patients and their families to inform and fully contribute to therapy development and prepare for clinical trials. By the end of the project, BATCure expects to have a lead therapy ready for a clinical trial and to have developed faster diagnostics suitable for presymptomatic testing and monitoring efficacy of new treatments.

Acknowledgment. BATCure consortium is funded under the EU Horizon2020 research innovation programme under grant agreement 666918. 